J. Clin. Chem. Clin. Biochem.

Vol. 19, 1981, pp. 1181-1187

\title{
Katalytische Konzentration, multiple Formen und Lektinaffinität von mikrosomalen Enzymen
menschlicher Gewebe
}

\author{
Lektine als Reagentien, II. Mitteilung
}

Von K. Lorentz

Aus dem Institut für Klinische Chemie (Direktor: Prof. Dr. K. Lorentz) der Medizinischen Hochschule Lübeck

(Eingegangen am 16. März/24. August 1981)

Herrn Prof. Dr. J. Kühnau zum 80. Geburtstag gewidmet

Zusammenfassung: Es wurden die katalytischen Konzentrationen, multiplen Formen und Lektinaffinitäten von Arylamidase, alkalischer Phosphatase, $\boldsymbol{\gamma}$-Glutamyltransferase, Cholinesterase und Arylesterase in partikelfreien Extrakten menschlicher Gewebe untersucht. Alle Enzyme bis auf Arylesterase reagierten intensiv mit den Lektinen aus Ricinus communis (Typ 120), Canavalia ensiformis, Triticum vulgare und Phaseolus vulgaris $\mathrm{S}$, während die Agglutinine aus Glycine max, Arachis hypogaea und Ulex europaeus kaum präzipitierten. Diese Reaktionen wurden stärker von der Herkunft als von der Art des Enzyms bestimmt. Da die Abspaltung von N-Acetylneuraminsäure die Fällung durch Triticum vulgare-Lektin immer verminderte und in Extrakten aus normalem Leberparenchym sogar aufhob, sind Sialoenzyme offenbar regelhaft bereits intrazellulär vorhanden. Abweichende Reaktionen von Enzymen aus intaktem und carcinomatösem Lebergewebe fanden sich nicht in den Seren entsprechender Patienten wieder. Dasselbe traf für die Muster multipler Formen zu. Die Ursachen dieser Unterschiede werden erörtert.

Catalytic concentration, multiple forms, and lectin affinity of microsomal enzymes from human tissues

Lectins as reagents, II.

Summary: We estimated the concentrations, multiple forms, and lectin binding of five microsomal enzymes in particle free extracts from human kidney, pancreas, jejunal mucosa, and normal and cancerous liver. While arylesterase markedly reacted only with concanavalin $A$, arylamidase, alkaline phosphatase, $\gamma$-glutamyltransferase, and cholinesterase were intensely precipitated by lectins from Ricinus communis 120, Canavalia ensiformis, Triticum vulgare and Phaseolus vulgaris S. Agglutinins from Glycine max, Arachis hypogaea and Ulex europaeus proved less effective. The reaction mainly depended on the origin of enzymes not on their species. Desialylation always decreased precipitation, and in extracts of normal liver parenchyma it even totally abolished precipitation, by Triticum vulgare lectin. Sialoenzymes therefore appear to be normal intracellular constituents. Differences between enżymes from normal and cancerous liver were not reflected by variant properties of the corresponding activities in sera. The same held true for multiple forms. The reasons for these differences are discussed.

\section{Einfuihrung}

Die Reaktion verschiedener Lektine mit mikrosomalen Enzymen im Serum zeigtè komplexe Muster, deren Deutung unsicher wạ und nur bei bösartigen Tumoren und bei chronisch entzündlichen Leberleiden diagnostische Hinweise gab (1). Daher untersuchten wir das Verhalten dieser Enzyme in den partikelfreien Rohextrakten jener Organe, die als Ursprungsorte der Aktivität im Serum gelten. Hierzu wurden von fünf mikrosomalen
Enzymen die katalytischen Konzentrationen, multiplen Formen und Lektinaffinitäten bestimmt.

\section{Material und Methoden}

Material

Alle Proben stammten aus identischen Arealen makroskopisch unauffälliger Organe: Lebergewebe aus dem rechten Lappen (zentral), Jejunummucosa vom proximalen Ende $(10 \mathrm{~cm}$ unter der flexura duodenojejunalis), Nierenrinde aus dem oberen Pol des Organs, Knochen vom Sternum an Costalgelenken und Pan- 
kreasparenchym aus der Mitte der Cauda pancreatis ${ }^{1}$ ). Die auch histologisch unveränderten Gewebsproben wurden, ebenso wie Excisate aus nicht nekrotischen Lebermetastasen, intraoperativ oder 10-24 h nach dem Tode entnommen, von bindegewebigen Anteilen befreit, in etwa $0,2 \mathrm{~g}$ wiegende Stücke zerschnitten und mit Natriumchlorid $155 \mathrm{mmol} / 1$ möglichst frei von Blut gespült. Knochen wurde mit einem Hammer zerschlagen und anschließend wie die anderen Gewebe mit 5 Tln. Triton X-100 $10 \mathrm{~g} / 1$ in Pufferlösung $C$ unter Eiskühlung $4 \times 10 \mathrm{~s}$ mittels Ultra-Turrax (Jahnke \& Kunkel, Staufen) und $1 \times 20 \mathrm{~s}$ mit einem Gaspistill nach Potter-Elvehjem homogenisiert und dann bei $-28^{\circ} \mathrm{C}$ eingefroren. Alle Homogenate tauten wir ohne nachweisbaren Aktivitätsverlust innerhalb eines Monats auf und zentrifugierten sie bei $0^{\circ} \mathrm{C} 60 \mathrm{~min}$ mit $100000 \times \mathrm{g}$. Durch diese Behandlung in Gegenwart eines Detergenz' gelangten mehr als 95\% der Enzymaktivität in den partikelfreien Überstand. Ein probatorischer Zusatz von Triton X-100 zu detergenzfreien Organextrakten bis zur verwendeten Endkonzentration von $8,3 \mathrm{~g} / 1$ veränderte weder die Affinität zu Lektinen noch die gemessene katalytische Konzentration der Enzyme.

Alle Reagentien und Lösungen entsprachen denen der I. Mitteilung (1), auch die Lektine. Den Konzentrationsangaben im Ansatz lagen die dort aufgefuhrten Molekulargewichte zugrunde.

Methoden

Wie bei den Untersuchungen mit Seren (1) wurden gleiche Tle. von partikelfreiem Extrakt und Pufferlösung gemischt und $2 \mathrm{~h}$ bei $25^{\circ} \mathrm{C}$ inkubiert. Zur Fällung wurden die verwendeten Lektine der Lösung $C$, bei Vorbehandlung mit Neuraminidase $\left(50000 \mathrm{E} / 1,16 \mathrm{~h}\right.$ bei $\left.25^{\circ} \mathrm{C}\right)$, die in Lösung $A$ enthalten war, dagegen der Lösung $B$ zugesetzt, um einen pH-Wert von 7,0-7,2 im Ansatz zu garantieren.

Lösung A (Acetat-Tris $250 \mathrm{mmol} / \mathrm{l}$, Calciumchlorid $20 \mathrm{mmol} / 1$, $\mathrm{pH} 5,0)$

Lösung B (Tris-Acetat $500 \mathrm{mmol} / \mathrm{l}$, Calciumchlorid $2 \mathrm{mmol} / \mathrm{l}$, Magnesiumchlorid $2 \mathrm{mmol} / \mathrm{l}, \mathrm{pH} 7,7$ )

Lösung C (Tris-Acetat $100 \mathrm{mmol} / \mathrm{l}$, Calciumchlorid $500 \mu \mathrm{mol} / \mathrm{l}$, Magnesiumchlorid $500 \mu \mathrm{mol} / \mathrm{l}$, Mangan(II)chlorid $10 \mu \mathrm{mol} / 1, \mathrm{pH} \mathrm{7,2)}$

In den nach Zentrifugation ( 3 min mit $12000 \times g$ ) der Ansätze resultierenden Überständen wurden die katalytischen Konzentrationen der Enzyme im Mikroverfahren (1) bei $25^{\circ} \mathrm{C}$ und ihre Verteilung bei diskelektrophoretischer Trennung (1) ermittelt. In den als Ausgangsmaterial verwendeten partikelfreien Extrakten bestimmten wir die Proteinkonzentration nach Richterich (2) mit Biuret-Reagenz gegen Probenleerwerte, die ebenso wie der Albuminstandard und der Reagenzleerwert die Tritonkonzentration der Proben aufwiesen.

Im einzelnen wurden folgende Bestimmungen und Verfahren benutzt:

Arylamidase, mikrosomal (EC 3.4.11.2) nach l.c. (3), alkalische Phosphatase (EC 3.1.3.1) nach 1.c. (4), $\gamma$-Glutamyltransferase (EC 2.3.2.2) nach 1.c. (5) und Cholinesterase (EC 3.1.1.8) nach 1.c. (6). Da, im Gegensatz zum Serum, Organextrakte außer Arylesterase weitere phenylacetatspaltende Carboxylesterasen (EC 3.1.1-) enthalten, wurde dieses Enzym (EC 3.1.1.2) in Gegenwart von Paraoxon $1 \mu \mathrm{mol} / \mathrm{l}$ nach l.c. (7) gemessen.

Die multiplen Formen der Enzyme wurden in Polyacrylamidgradientengelen (8) nach 1.c. (1) dargestellt, die der $\gamma$-Glutamyltransferase nach Patel \& O'Gorman (9).

Zum Vergleich der spezifischen Aktivitäten in normalem und maligne in entartetem Lebergewebe diente der WilcoxonRangsummen-Test für nicht korrelierte Stichproben. Ein Zusammenhang der Meßdaten verschiedener Enzyme im selben Organ wurde mit der Rangkorrelation nach Spearman geprüft.

1) Herrn Prof. Dr. A. Gropp (Direktor des Instituts für Pathologie der Med. Hochschule Lübeck),

Herrn Piof. Dr. O. Pribilla (Direktor des Instituts für Rechtsmedizin der Med. Hochschule Lübeck) und

Herrn Prof. Dr. J. Durst (Chefarzt der Chirurgischen Klinik des Städt. Krankenhauses Lübeck) danke ich für die Überlassung der Proben.

\section{Ergebnisse}

\section{Spezifische Aktivität}

Da autolytisch verändertes Material verworfen wurde, unterschieden sich die katalytischen Konzentrationen der solubilisierten Enzyme aus intraoperativ und post mortem entnommenen Proben nicht voneinander. Eine Ubersicht aller Ergebnisse bietet Tabelle 1. Vergleicht man die spezifische Aktivität der verschiedenen Enzyme jeweils in derselben Gewebsprobe, so zeigen ihre Werte zumeist kein konstantes Muster. Daher enthält Tabelle 2 nur statistisch signifikante Korrelationen zwischen bestimmten Aktivitäten.

Arylamidase und Cholinesterase wiesen die engsten Verteilungsbereiche auf, während die spezifische Aktivität der Arylesterase am stärksten streute. Vergleiche mit den Ergebnissen anderer Autoren (Leber: 1.c. (10-14); Niere: 1.c. $(12,15,16)$; Jejunum: 1.c. $(17,18)$; Knochen: 1.c. (19)) sind wegen der unterschiedlichen Testverfahren und Meßtemperaturen problematisch. Die hier mitgeteilten Werte lassen sich hingegen mit den katalytischen Konzentrationen im Serum in Bezug setzen. Auffällig war dabei die gegenüber dem Serum geringe Aktivität beider Esterasen.

Die spezifischen Aktivitäten korrelierten am häufigsten und engsten im Metastasengewebe. Dies ist offenbar ein Ausdruck der Entdifferenzierung, da hier, im Gegensatz zu reifenden Epithelzellen des gesunden Gewebes, Altersunterschiede einzelner Zellen mit ihrem Wandel der Enzymgarnitur weitgehend fehlen. Gleichwohl konnten die Aktivitätsmuster dem histologischen Typ der Metastasen nicht zugeordnet werden. Dies galt auch für die Exportproteine Cholinesterase und Arylesterase, als deren Herkunftsort der normale Hepatocyt zu gelten hat. Andererseits zeigte kein untersuchtes Enzym einen statistisch signifikanten Unterschied seiner spezifischen Aktivität zwischen Metastasen- und normalem Lebergewebe. Weiterhin fehlte im neoplastisch veränderten Parenchym ein typisches Enzymmuster.

\section{Multiple Formen}

Bei der elektrophoretischen Trennung entsprachen Anzahl und Reihenfolge der Fraktionen im Gradientengel weitgehend den Ergebnissen eigener Untersuchungen mit Stufengelen an alkalischer Phosphatase (20) und Arylamidase (21) aus Rohhomogenaten. Sie stimmten auch mit Beobachtungen an gereinigter alkalischer Phosphatase (17) und $\gamma$-Glutamyltransferase (13) überein. Die Zusammenfassung aller Zymogramme (Tab. 3) ließ eine - gegenüber intaktem Lebergewebe - deutliche Verminderung der multiplen Formen aller fünf Ënzyme bei maligner Entartung erkennen. Dağegen waren die hier nicht aufgeführten - Laufstrecken der schnellsten Fraktionen bei beiden Geweben identisch und wurden durch Vorbehandlung mit Neuraminidase in gleichem Ausmaß verlangsamt. Im übrigen ergaben sích, unab- 

Tab. 1. Konzentration (U/g Protein) fünf mikrosomaler Enzyme im partikelfreien Überstand von 17 Rohextrakten menschlicher
Gewebe.

Bereich (jeweils obere Reihe), Mittelwert und Standardabweichung (darunter) und Verteilungstyp:
normal, ${ }_{\text {lognormal, }}^{+}$nicht nachweisbar

\begin{tabular}{|c|c|c|c|c|c|c|}
\hline Gewebe & \multicolumn{2}{|c|}{$\begin{array}{l}\text { Arylamidase } \\
\mathrm{U} / \mathrm{g} \\
\end{array}$} & $\begin{array}{l}\text { Alkalische } \\
\text { Phosphatase } \\
\text { U/g }\end{array}$ & $\begin{array}{l}\boldsymbol{\gamma} \text {-Glutamyl- } \\
\text { transferase } \\
\mathrm{U} / \mathrm{g}\end{array}$ & $\begin{array}{l}\text { Cholinesterase } \\
\mathrm{U} / \mathrm{g}\end{array}$ & $\begin{array}{l}\text { Arylesterase } \\
\mathrm{U} / \mathrm{g}\end{array}$ \\
\hline Leber & $\begin{array}{l}2,35- \\
8,52 \pm\end{array}$ & $\begin{array}{r}-22,2^{\circ} \\
\pm \quad 1,94\end{array}$ & $\begin{array}{c}8,78-141^{*} \\
39,2 \pm 32,2\end{array}$ & $\begin{array}{l}13,9-105^{*} \\
50,2 \pm 31,6\end{array}$ & $\begin{array}{r}4,40-48,3^{\bullet} \\
18,1 \pm 2,58\end{array}$ & $32,2-1570^{+}$ \\
\hline $\begin{array}{l}\text { Leber- } \\
\text { metastasen }^{1} \text { ) }\end{array}$ & $\begin{array}{l}4,65- \\
15,0 \pm\end{array}$ & $\begin{array}{l}-53,2^{*} \\
\pm \quad 12,5\end{array}$ & $4,92-203^{+}$ & $\begin{array}{l}28,2-154^{*} \\
89,6-81,6\end{array}$ & $\begin{array}{l}4,74-43,9^{\bullet} \\
9,90 \pm 4,22\end{array}$ & $31,9-968^{+}$ \\
\hline Niere (Cortex) & $\begin{array}{l}10,8 \\
146 \quad \pm\end{array}$ & $\begin{array}{l}-273^{*} \\
\pm \quad 77,6\end{array}$ & $\begin{aligned} 8,47 & -55,5^{*} \\
28,4 & \pm 12,6\end{aligned}$ & $\begin{array}{c}43,6-1490^{*} \\
689 \pm 431\end{array}$ & $\begin{array}{l}1,93-21,3^{*} \\
8,65 \pm 4,63\end{array}$ & $33,2-166^{+}$ \\
\hline Pankreas & $\begin{array}{r}6,50 \\
24,7 \pm\end{array}$ & $\begin{array}{l}-47,7^{*} \\
\pm 14,4\end{array}$ & $2,30-138^{+}$ & $4,40-851^{+}$ & $\begin{array}{r}9,11-35,1^{*} \\
18,3 \pm 5,34\end{array}$ & $10,7-304$ \\
\hline Jejunummucosa & $\begin{array}{l}16,5- \\
48,3 \pm\end{array}$ & $\begin{array}{l}-129^{*} \\
\pm \quad 30,4\end{array}$ & $34,9-685^{+}$ & $\begin{array}{l}13,2-83,7^{*} \\
34,7 \pm 18,5\end{array}$ & $\begin{array}{l}23,4-78,6^{*} \\
54,1 \pm 19,8\end{array}$ & $\begin{array}{lr}17,6- & 201^{\bullet} \\
77,3 \pm & 56,6\end{array}$ \\
\hline Knochen & - & & $6,30-247^{+}$ & - & - & - \\
\hline
\end{tabular}

1) Histologischer Typ des Metastasengewebes: Entdifferenziertes Carcinom der Gallenwege 4, kleinzelliges Bronchialcarcinom 5 , Magencarcinom vom intestinalen Typ 3, Adenocarcinom der Mamma 2, verhornendes Plattenepithelcarcinom (vom Collum uteri) 2 .

Tab. 2. Korrelation der katalytischen Konzentration mikrosomaler Enzyme im partikelfreien Uberstand von 17 Rohextrakten menschlicher Gewebe. $\rho=$ Korrelationskoeffizient, $\quad \mathrm{p}=$ Irrtumswahrscheinlichkeit (nach Spearman).

\section{Organ}

Kenngrößen

Leber

Arylamidase - Alkal. Phosphatase

Arylämidàse $-\gamma$-Glutamyltransferase

Cholinesterase - Arylesterase

\section{Lebermetastasen}

Arylamidase - Alkal. Phosphatase Arylamidase $-\gamma$-Glutamyltransferase

Arylamidase - Arylesterase

Alkal. Phosphatase $-\gamma$-Ğlutamyltransferase

Alkal. Phosphatase - Arylesterase

Cholinesterase - Arylesterase

$\rho \quad \mathrm{p}$

p

\section{Pankreas}

Arylamidase $-\boldsymbol{\gamma}$-Glutamyltransferase

$<0,01$

Niere (Cortex)

Arylamidase $-\boldsymbol{\gamma}$-Glutamyltransferase

Cholinesterase - Arylesterase

$0,590<0,05$

$0,908<0,01$

hängig vom Lebèns- und Entnahmealter, zwișchen verschiedenen Proben desselben Organs nur geringe Unterschiede durch das gelegentliche Auftreten von langsam wandernden Minorbanden, die wenigè als 30\% der Aktivität von Hauptfraktionen enthielten.

\section{Lektinaffinität}

In der voraufgegangenen Studie an Seren (1) warẹn nur sieben von zehn geprüften Lektinen zur Unterscheidung
Tab. 3. Multiple Formen von fünf mikrosomalen Enzymen in partikelfreien Überständen von Rohextrakten menschlicher Organe. Angabe der Bandenzahl bei Elektrophorese im Polyacrylamidgradientengel, ()$=$ inkonstantes Auftreten kathodischer Minorfraktionen (mit weniger als $30 \%$ Aktivität der Hauptfraktionen).

\begin{tabular}{|c|c|c|c|c|c|}
\hline Gewebe & $\begin{array}{l}\text { Aryl- } \\
\text { amidase }\end{array}$ & $\begin{array}{l}\text { Alka- } \\
\text { lische } \\
\text { Phos- } \\
\text { phatase }\end{array}$ & $\begin{array}{l}\gamma \text {-Glut- } \\
\text { amyl- } \\
\text { trans- } \\
\text { ferase }\end{array}$ & $\begin{array}{l}\text { Cholin- } \\
\text { esterase }\end{array}$ & $\begin{array}{l}\text { Aryl- } \\
\text { esterase }\end{array}$ \\
\hline Leber & $2(-3)$ & 2 & 1 & $3(-4)$ & 6 \\
\hline $\begin{array}{l}\text { Leber- } \\
\text { metastasen }\end{array}$ & $1(-2)$ & 1 & 1 & 3 & 2 \\
\hline $\begin{array}{l}\text { Niere } \\
\text { (Cortex) }\end{array}$ & 2 & 2 & 1 & 2 & 4 \\
\hline Pankreas & 2 & 2 & 3 & 1 & $1(-2)$ \\
\hline $\begin{array}{l}\text { Jejunum- } \\
\text { mucosa }\end{array}$ & $2(-3)$ & 2 & 1 & 2 & 1 \\
\hline Knochen & 1 & 2 & - & - & - \\
\hline
\end{tabular}

von Gesunden, Leberkranken und Carcinomträgern geeignet. Daher verwendeten wir diese, $z$. T. schlecht löslichen, Agglutinine in höchstmöglicher Konzentration. Die Summe der Aktivität in Uberstand und Niederschlag entsprach den Ausgangswerten.

Arachis hypogaea-Lektin $10 \mu \mathrm{mol} / 1$ und Ulex europaeusLektin (Typ I) $50 \mu \mathrm{mol} / 1$ fällten keines der untersuchten Enzyme aus den partikelfreien Organextrakten, so daß dort zugängliche Galaktose- und Fucosereste fehlen dürften. Arylesterase reagierte nur mit Concanavalin A, offenbar infolge exponierter Mannoseeinheiten in ihrem Kohlenhydratanteil. Während mit anderen Lektinen nach der Inkubation 0,92-0,99 der Ausgangsaktivität $(=1,0)$ im Uberstand verblieben, lauteten die Werte $(\bar{x} \pm s$, $\mathrm{n}=12$ ) nach Präzipitation mit Concanavalin A $80 \mu \mathrm{mol} / 1$ 
$0,216 \pm 0,086$ bei normalem Lebergewebe, $0,552 \pm 0,147$ in Metastasen der Leber, $0,363 \pm 0,163$ in der Nierenrinde, $0,609 \pm 0,260 \mathrm{im}$ Pankreas und 0,570 $\pm 0,146$ in der Jejunummucosa.

Eine Synopsis über die Lektinaffinität der anderen Enzyme zeigt das abweichende Verhalten von Cholinesterase (Tab. 4). Ordnet man die Ergebnisse nach Organen, so reagieren die Aktivitäten der Jejunummucosa, Cholinesterase ausgenommen, am schwächsten mit Lektinen. Betrachtet man die Resultate im Hinblick auf den Einfluß der Agglutinine, so fällt das Ricinus communis 120-Lektin trotz geringster Konzentration am wirkungsvollsten die Enzyme aller Organe, wiederum mit Ausnahme der Cholinesterase. Geht man von den Enzymen aus, dann nimmt Cholinesterase eine Sonderstellung ein: In Extrakten aus Jejunumschleimhaut wird sie von den vier Lektinen aus Canavalia ensiformis, Triticum vulgare, Phaseolus vulgaris $\mathrm{S}$ und Ricinus communis 120 deutlich stärker, in den Extrakten aus normalem und carcinomatösem Lebergewebe jedoch wesentlich schwächer gefällt als die anderen Enzyme. Auch bei der Untersuchung von Nieren- und Pankreasgewebe war Cholinesterase gegenüber den genannten Agglutininen mit Ausnahme von Concanavalin A weitaus resistenter als die übrigen Aktivitäten.

Die Behandlung mit Neuraminidase veränderte in keinem Gewebe, trotz des abweichenden pH-Wertes während der Inkubation, die ursprünglich gemessene Enzymkonzentration. Nur die alkalische Phosphatase des Knochengewebes nahm in ihrer Aktivität um den Faktor $1,29 \pm 0,22 \mathrm{zu}$. Im einzelnen waren folgende Reaktionen hervorzuheben:

Glycine max-Lektin $20 \mu \mathrm{mol} / 1$ war wenig wirksam und fälte nur in Pankreasextrakten. Erst nach Vorbehandlung mit Neuraminidase reagierten alle Enzyme aus Metastasengewebe, sowie die alkalische Phosphatase, $\gamma$-Glutamyltransferase und Cholinesterase der Niere. Dagegen waren Aktivitäten aus den Extrakten von normalem Leberparenchym und Jejunummucosa auch nach Abspaltung von Sialinsäure nicht zu fällen. Die Reaktionen verhielten sich also eher organ- als enzymspezifisch.

Während mit diesem Agglutinin ein Nachweis von Galaktose- und/oder N-Acetylgalaktosaminresten kaum gelang, präzipitierte Phaseolus vulgaris S-Lektin, das bei ähnlichem Molekulargewicht eine identische Zuckerspezifität aufweist, schon bei gleicher Konzentration wesentlich stärker und bei $40 \mu \mathrm{mol} / 1 \mathrm{sehr}$ effektiv (s. Tab. 4). Auch sein Verhalten war organtypisch, da, abgesehen von der Cholinesterase, die der Niere entstammenden Aktivitäten am besten gefällt wurden.

Da Agglutinin 120 aus Ricinus communis ebenfalls bei übereinstimmendem Molekulargewicht - gegen Galaktosereste gerichtet ist, war ein ähnliches Reaktionsmuster zu erwarten. Tatsächlich verhielten sich beide Agglutinine weitgehend gleich. Größere Abwei- chungen traten nur bei der Reaktion von Arylamidase, alkalischer Phosphatase und $\gamma$-Glutamyltransferase aus intaktem und maligne entartetem Leberparenchym auf. Sie verlief mit Ricinus communis 120-Lektin intensiver. Uberhaupt ist dieses Agglutinin optimal zur Fällung aller alkalischen Phosphatasen mit Ausnahme der intestinalen Aktivität geeignet.

Uberraschenderweise trat nach Abspaltung von endständiger N-Acetylneuraminsäure bei Leberextrakten statt der erwarteten Reaktionszunahme eine - teilweise eindrucksvolle - Verminderung der Lektinaffinität ein. Sie wurde beim Agglutinin aus Triticum vulgare ausnahmslos beobachtet und führte bei den Enzymen der Leber sogar zur Aufhebung der Reaktion. Da dieses Lektin neben N-Acetylglucosamin- auch N-Acetylgalaktosamin- (22) und vor allem N-Acetylneuraminsäurereste $(22,23,24)$ erfaß̧t, bedeutet der Verlust der Bindungsfähigkeit, daß in den Enzymen hepatischer Herkunft Sialinsäure als einziger Partner erscheint, in allen anderen aber zumindest als obligater Bestandteil vorkommen muß. So war das Fehlen von Sialoenzymen in allen untersuchten Geweben auszuschließen.

Problematischer erscheint die nach Einwirkung von Neuraminidase abnehmende Reaktion mit anderen Lektinen. Bei Concanavalin A betraf sie selektiv die vier Enzyme aus normaler Leber, bei Ricinus communis 120- und Phaseolus vulgaris S-Lektin ebenfalls die hepatischen Aktivitäten, Cholinesterase ausgenommen. Weiterhin wurde mit dem zuletzt genannten Agglutinin eine verminderte Reaktion bei sämtlichen alkalischen Phosphatasen und allen $\gamma$-Glutamyltransferasen (außer bei der intestinalen) beobachtet. Durch Mitführen nicht hydrolysierter 4-Nitrophenylglucoside konnte eine Verunreinigung der Ansätze durch andere Glucosidasen mit konsekutiver Abspaltung von Zuckern ausgeschlossen werden. Auch eine Modifikation des Bindungsverhaltens durch autolytisch aus den Proben freigesetzte Proteasen erscheint unwahrscheinlich, da wegen der langen Inkubation bei $\mathrm{pH} 5$ ein Ansatz ohne Neuraminidase als Bezugswert $(=1,0)$ für diese Versuche verwendet wurde. In keinem Fall verstärkte die Vorinkubation mit Neuraminidase die Reaktion von Enzymen mit Concanavalin A, so daß eine Bindungshemmung durch terminale Sialinsäurereste, wie sie für $\gamma$-Glutamyltransferasen angenommen wurde (25), entfallt.

Die Muster der multiplen Formen erfuhren durch die Behandlung mit Lektinen keine qualitative Veränderung, wenn man von der Verzögerung einzelner Fraktionen durch Bildung höhermolekularer Protein-LektinKomplexe absieht. In den angewandten Konzentrationen reduzierte das Agglutinin 120 aus Ricinus communis die Intensität aller Banden am deutlichsten, wie aus den Untersuchungen der Gesamtaktivität zü erwarten war. Eine selektive Elimination einzelner Fraktionen fand nicht statt. Vorherige Hydrolyse mit Neuraminidase veränderte die Muster der multiplen Formen nicht. 
Tab. 4. Relative Aktivität in den Uberständen partikelfreier Extrakte von menschlichen Organen nach Fällung mit Lektinen, bezogen auf die ursprüngliche katalytische Konzentration $(1,0)$ ohne (jeweils obere Reihe) oder mit Neuraminidasevorbehandlung Methodik.
Meihe). Mittelwert und Standardabweichung aus der Untersuchung von 12 verschiedenen Proben, Vorgehen s.

\begin{tabular}{|c|c|c|c|c|c|}
\hline & $\begin{array}{l}\text { Canavalia } \\
\text { ensiformis }\end{array}$ & Triticum vulgare & $\begin{array}{l}\text { Phaseolus } \\
\text { vulgaris S }\end{array}$ & $\begin{array}{l}\text { Ricinus } \\
\text { communis } 120\end{array}$ & Glycine $\max$ \\
\hline & $80 \mu \mathrm{mol} / 1$ & $80 \mu \mathrm{mol} / 1$ & $40 \mu \mathrm{mol} / 1$ & $15 \mu \mathrm{mol} / 1$ & $20 \mu \mathrm{mol} / 1$ \\
\hline & Leber & & & & \\
\hline Arylamidase & $\begin{array}{l}0,110 \pm 0,106 \\
0,210 \pm 0,195\end{array}$ & $\begin{array}{l}0,215 \pm 0,112 \\
0,940 \pm 0,180\end{array}$ & $\begin{array}{l}0,274 \pm 0,152 \\
0,810 \pm 0,220\end{array}$ & $\begin{array}{l}0,071 \pm 0,069 \\
0,580 \pm 0,120\end{array}$ & $\begin{array}{l}1,020 \pm 0,030 \\
0,865 \pm 0,105\end{array}$ \\
\hline Alkalische Phosphatase & $\begin{array}{l}0,196 \pm 0,1.02 \\
0,463 \pm 0,228\end{array}$ & $\begin{array}{l}0,126 \pm 0,107 \\
0,992 \pm 0,063\end{array}$ & $\begin{array}{l}0,308 \pm 0,112 \\
0,390 \pm 0,131\end{array}$ & $\begin{array}{l}0,038 \pm 0,024 \\
0,161 \pm 0,098\end{array}$ & $\begin{array}{l}0,975 \pm 0,085 \\
0,967 \pm 0,077\end{array}$ \\
\hline$\gamma$-Glutamyltransferase & $\begin{array}{l}0,136 \pm 0,075 \\
0,576 \pm 0,219\end{array}$ & $\begin{array}{l}0,181 \pm 0,076 \\
0,979 \pm 0,051\end{array}$ & $\begin{array}{l}0,402 \pm 0,210 \\
0,910 \pm 0,040\end{array}$ & $\begin{array}{l}0,067 \pm 0,043 \\
0,740 \pm 0,312\end{array}$ & $\begin{array}{l}1,002 \pm 0,018 \\
0,959 \pm 0,043\end{array}$ \\
\hline \multirow[t]{2}{*}{ Cholinesterase . } & $\begin{array}{l}0,245 \pm 0,177 \\
0,510 \pm 0,321\end{array}$ & $\begin{array}{l}0,680 \pm 0,128 \\
1,030 \pm 0,082\end{array}$ & $\begin{array}{l}0,768 \pm 0,157 \\
0,802 \pm 0,176\end{array}$ & $\begin{array}{l}0,775 \pm 0,201 \\
0,833 \pm 0,234\end{array}$ & $\begin{array}{l}0,996 \pm 0,265 \\
1,033 \pm 0,041\end{array}$ \\
\hline & Lebermetastasen & & & & \\
\hline Arylamidase & $\begin{array}{l}0,469 \pm 0,100 \\
0,393 \pm 0,150\end{array}$ & $\begin{array}{l}0,207 \pm 0,196 \\
0,792 \pm 0,180\end{array}$ & $\begin{array}{l}0,246 \pm 0,220 \\
0,266 \pm 0,213\end{array}$ & $\begin{array}{l}0,173 \pm 0,220 \\
0,079 \pm 0,052\end{array}$ & $\begin{array}{l}0,990 \pm 0,044 \\
0,482 \pm 0,147\end{array}$ \\
\hline Alkalische Phosphatase & $\begin{array}{l}0,390 \pm 0,184 \\
0,252 \pm 0,163\end{array}$ & $\begin{array}{l}0,066 \pm 0,062 \\
0,716 \pm 0,221\end{array}$ & $\begin{array}{l}0,234 \pm 0,123 \\
0,462 \pm 0,173\end{array}$ & $\begin{array}{l}0,071 \pm 0,068 \\
0,000\end{array}$ & $\begin{array}{l}1,025 \pm 0,046 \\
0,351 \pm 0,069\end{array}$ \\
\hline$\gamma$-Glutamyltransferase & $\begin{array}{l}0,469 \pm 0,100 \\
0,439 \pm 0,120\end{array}$ & $\begin{array}{l}0,138 \pm 0,090 \\
0,817 \pm 0,081\end{array}$ & $\begin{array}{l}0,319 \pm 0,186 \\
0,428 \pm 0,213\end{array}$ & $\begin{array}{l}0,118 \pm 0,092 \\
0,054 \pm 0,029\end{array}$ & $\begin{array}{l}0,983 \pm 0,051 \\
0,640 \pm 0,177\end{array}$ \\
\hline \multirow[t]{2}{*}{ Cholinesterase } & $\begin{array}{l}0,305 \pm 0,197 \\
0,296 \pm 0,204\end{array}$ & $\begin{array}{l}0,314 \pm 0,152 \\
0,845 \pm 0,083\end{array}$ & $\begin{array}{l}0,382 \pm 0,183 \\
0,402 \pm 0,196\end{array}$ & $\begin{array}{l}0,448 \pm 0,196 \\
0,391 \pm 0,218\end{array}$ & $\begin{array}{l}0,860 \pm 0,170 \\
0,416 \pm 0,191\end{array}$ \\
\hline & Niere (Cortex) & & & & \\
\hline Arylamidase & $\begin{array}{l}0,259 \pm 0,120 \\
0,322 \pm 0,108\end{array}$ & $\begin{array}{l}0,165 \pm 0,112 \\
0,879 \pm 0,120\end{array}$ & $\begin{array}{l}0,074 \pm 0,057 \\
0,110 \pm 0,032\end{array}$ & $\begin{array}{l}0,047 \pm 0,031 \\
0,070 \pm 0,042\end{array}$ & $\begin{array}{l}0,920 \pm 0,104 \\
0,980 \pm 0,090\end{array}$ \\
\hline Alkalische Phosphatase & $\begin{array}{l}0,605 \pm 0,109 \\
0,679 \pm 0,141\end{array}$ & $\begin{array}{l}0,203 \pm 0,094 \\
0,866 \pm 0,282\end{array}$ & $\begin{array}{l}0,070 \pm 0,051 \\
0,273 \pm 0,147\end{array}$ & $\begin{array}{l}0,070 \pm 0,049 \\
0,052 \pm 0,031\end{array}$ & $\begin{array}{l}0,917 \pm 0,090 \\
0,413 \pm 0,081\end{array}$ \\
\hline$\gamma$-Glutamyltransferase & $\begin{array}{l}0,960 \pm 0,075 \\
0,848 \pm 0,091\end{array}$ & $\begin{array}{l}0,470 \pm 0,143 \\
0,939 \pm 0,134\end{array}$ & $\begin{array}{l}0,161 \pm 0,078 \\
0,354 \pm 0,119\end{array}$ & $\begin{array}{l}0,286 \pm 0,101 \\
0,061 \pm 0,033\end{array}$ & $\begin{array}{l}1,008 \pm 0,028 \\
0,709 \pm 0,066\end{array}$ \\
\hline \multirow[t]{2}{*}{ Cholinesterase } & $\begin{array}{l}0,325 \pm 0,131 \\
0,365 \pm 0,152\end{array}$ & $\begin{array}{l}0,314 \pm 0,109 \\
0,843 \pm 0,112\end{array}$ & $\begin{array}{l}0,313 \pm 0,105 \\
0,362 \pm 0,130\end{array}$ & $\begin{array}{l}0,394 \pm 0,166 \\
0,505 \pm 0,178\end{array}$ & $\begin{array}{l}0,968 \pm 0,135 \\
0,472 \pm 0,129\end{array}$ \\
\hline & Pankreas & & & & \\
\hline Arylamidase & $\begin{array}{l}0,538 \pm 0,180 \\
0,458 \pm 0,124\end{array}$ & $\begin{array}{l}0,397 \pm 0,243 \\
0,848 \pm 0,224\end{array}$ & $\begin{array}{l}0,259 \pm 0,148 \\
0,268 \pm 0,137\end{array}$ & $\begin{array}{l}0,169 \pm 0,056 \\
0,196 \pm 0,088\end{array}$ & $\begin{array}{l}0,639 \pm 0,145 \\
0,675 \pm 0,150\end{array}$ \\
\hline Alkalische Phosphatase & $\begin{array}{l}0,484 \pm 0,159 \\
0,409 \pm 0,138\end{array}$ & $\begin{array}{l}0,127 \pm 0,090 \\
0,728 \pm 0,103\end{array}$ & $\begin{array}{l}0,126 \pm 0,079 \\
0,358 \pm 0,127\end{array}$ & $\begin{array}{l}0,100 \pm 0,069 \\
0,058 \pm 0,026\end{array}$ & $\begin{array}{l}0,757 \pm 0,166 \\
0,533 \pm 0,204\end{array}$ \\
\hline$\gamma$-Glutạmyltransferase & $\begin{array}{l}0,546 \pm 0,260 \\
0,504 \pm 0,242\end{array}$ & $\begin{array}{l}0,276 \pm 0,223 \\
0,773 \pm 0,187\end{array}$ & $\begin{array}{l}0,319 \pm 0,209 \\
0,440 \pm 0,223\end{array}$ & $\begin{array}{l}0,119 \pm 0,044 \\
0,060 \pm 0,038\end{array}$ & $\begin{array}{c}0,702 \pm 0,263 \\
0,819 \pm 0,214\end{array}$ \\
\hline \multirow[t]{2}{*}{ Cholinesterase } & $\begin{array}{l}0,411 \pm 0,166 \\
0,563 \pm 0,181\end{array}$ & $\begin{array}{l}0,377 \pm 0,191 \\
0,788 \pm 0,100\end{array}$ & $\begin{array}{l}0,419 \pm 0,179 \\
0,470 \pm 0,243\end{array}$ & $\begin{array}{l}0,492 \pm 0,184 \\
0,580 \pm 0,137\end{array}$ & $\begin{array}{l}0,864 \pm 0,266 \\
0,662 \pm 0,291\end{array}$ \\
\hline & Jejunummucosa & & & & \\
\hline Arylamidase & $\begin{array}{l}0,815 \pm 0,150 \\
0,670 \pm 0,172\end{array}$ & $\begin{array}{l}0,548 \pm 0,370 \\
0,832 \pm 0,262\end{array}$ & $\begin{array}{l}0,255 \pm 0,132 \\
0,317 \pm 0,151\end{array}$ & $\begin{array}{l}0,395 \pm 0,195 \\
0,361 \pm 0,182\end{array}$ & $\begin{array}{l}0,905 \pm 0,184 \\
0,921 \pm 0,161\end{array}$ \\
\hline Alkälische Phosphatase & $\begin{array}{l}0,585 \pm 0,189 \\
0,562 \pm 0,158\end{array}$ & $\begin{array}{l}0,467 \pm 0,264 \\
0,918 \pm 0,212\end{array}$ & $\begin{array}{l}0,340 \pm 0,185 \\
0,740 \pm 0,208\end{array}$ & $\begin{array}{l}0,450 \pm 0,267 \\
0,375 \pm 0,237\end{array}$ & $\begin{array}{l}0,991 \pm 0,040 \\
0,973 \pm 0,062\end{array}$ \\
\hline$\gamma$-Glutamyltransferase & $\begin{array}{l}0,595 \pm 0,134 \\
0,399 \pm 0,111\end{array}$ & $\begin{array}{l}0,320 \pm 0,171 \\
0,853 \pm 0,152\end{array}$ & $\begin{array}{l}0,315 \pm 0,156 \\
0,382 \pm 0,162\end{array}$ & $\begin{array}{l}0,273 \pm 0,107 \\
0,312 \pm 0,097\end{array}$ & $\begin{array}{l}1,014 \pm 0,028 \\
0,900 \pm 0,092\end{array}$ \\
\hline \multirow[t]{2}{*}{ Cholinesterase } & $\begin{array}{l}0,135 \pm 0,104 \\
0,115 \pm 0,092\end{array}$ & $\begin{array}{l}0,074 \pm 0,048 \\
0,494 \pm 0,218 \\
.\end{array}$ & $\begin{array}{l}0,113 \pm 0,100 \\
0,208 \pm 0,152\end{array}$ & $\begin{array}{l}0,115 \pm 0,043 \\
0,230 \pm 0,138\end{array}$ & $\begin{array}{l}0,971 \pm 0,102 \\
0,955 \pm 0,141\end{array}$ \\
\hline & Knochen & & & & \\
\hline Alkalische Phoșphatase & $\begin{array}{l}0,492 \pm 0,215 \\
0,530 \pm 0,242\end{array}$ & $\begin{array}{l}0,215 \pm 0,113 \\
0,763 \pm 0,272\end{array}$ & $\begin{array}{l}0,102 \pm 0,078 \\
0,314 \pm 0,117\end{array}$ & $\begin{array}{l}0,044 \pm 0,028 \\
0,092 \pm 0,038\end{array}$ & $\begin{array}{l}0,980 \pm 0,022 \\
0,621 \pm 0,312\end{array}$ \\
\hline
\end{tabular}




\section{Diskussion}

Die Ergebnisse dieser Studie entsprachen der Bindung. gereinigter Glykoenzyme durch trägergebundene Lektine. So stimmten die Reaktionen von $\gamma$-Glutamyltransferase $(26,27)$ und alkalischer Phosphatase $(28)$ gut mit unseren Daten überein. Dasselbe gilt für Befunde von Huseby (29) mit gereinigter $\boldsymbol{\gamma}$-Glutamyltransferase aus Leber, Niere und Pankreas, gefällt mit gelösten Lektinen. Demnach reagieren die in hohem Überschuß vorliegenden Lektine auch in Gegenwart anderer Glykoproteine stöchiometrisch mit den Enzymen des partikelfreien Überstandes. Ähnlich gut korrespondierte die Fällung von alkalischer Phosphatase durch Agglutinin 120 aus Ricinus communis mit dem Galaktosegehalt der hepatischen und der intestinalen Aktivität (17). Dagegen zeigte von den Arylamidasen aus Leber, Niere und Pankreas das Leberenzym nur eine Fällung mit Concanavalin A (30) und präzipitierte mit den Lektinen aus Triticum vulgare und Phaseolus vulgaris $\mathrm{S}-\mathrm{im}$ Gegensatz zu unseren Beobachtungen - erst nach Inkubation mit Neuraminidase.

Für die hieraus abzuleitende sterische Reaktionshemmung durch endständige N-Acetylneuraminsäure sprachen nur die nach ihrer Abspaltung zunehmende Fällung durch Agglutinin 120 aus Ricinus communis und - in geringerem Ausmaß - Glycine max-Lektin. Beide sind gegen Galaktose, die vorher in präterminaler Position war, und nunmehr frei zugänglich ist, gerichtet. Eine ähnliche Reaktionszunahme mit den Lektinen aus Canavalia ensiformis und Phaseolus vulgaris S erscheint, trotz gegenteiliger Befunde mit Coeruloplasmin (31), danach auch theoretisch ausgeschlossen, da die ihnen entsprechenden Mannose- oder Glucose- bzw. N-Acetylgalaktosaminreste oder die Sequenz Galaktose $(\beta-1,4)$ $\mathrm{N}$-Acetylglucosamin ( $\beta$-1,2)-Mannose (32) durch die Entfernung des Sialylrestes nicht exponiert werden.

Vielmehr beobachteten wir eine stärkere Concanavalin A-Reaktion mit Sialoenzymen, vor allem bei Leberextrakten. Dies entspricht Mitteilungen über die alkalische Phosphatase des Dünndarms $(33,34,35)$ und eigenen Untersuchungen an Seren (1). Die Ursache dieses Verhaltens bleibt unklar. Eine Abnahme der Lektinbindung, die Shaw et al. (12) durch tryptische Behandlung von $\gamma$ Glutamyltransferase bei unveränderter Aktivität erzielten, ist wegen der mitgeführten Kontrollansätze ohne Neuraminidase jedoch auszuschließen.
Obwohl in den partikelfreien Extrakten eher ein organtypisches als ein enzymspezifisches Verhalten der Aktivitäten, vor allem der Leberenzyme vor und nach Einwirkung von Neuraminidase vorlag, bestand kein Zusammenhang zwischen der Lektinbindung derselben Enzyme in Seren von Gesunden und Carcinomträgern und den Extrakten aus normalem und entartetem Lebergewebe.

Ähnliches gịlt für die Muster der multiplen Formen. Den komplexen Zymogrammen des Serums standen nur wenige und gleichermaßen lektinempfindliche Fraktionen in den Extrakten gegenüber. Da $\gamma$-Glutamyltransferase in Pherogrammen von Seren in bis zu drei Zonen vorkommt (36), die durch Interaktion des Enzyms mit Lipiden und Proteinen entstehen (37), ist deren Veränderung allein durch die Gegenwart von Triton denkbar (29). Dem widerspricht hingegen die Tatsache, daß nur 3\% der $\boldsymbol{\gamma}$-Glutamyltransferaseaktivität von Serum in hydrophober Bindung vorliegen und durch Triton freizusetzen sind (38). Vielmehr reflektiert die $\boldsymbol{\gamma}$-Glutamyltransferase des Serums zu über 84\% das durch Papainbehandlung lösliche Enzym der Leber (38). Da die Aktivitäten aus Serum, Leber und Niere immunologische Identität aufweisen (39), sich aber deutlich in der Ladung (39) und weniger im Molekulargewicht (38) unterscheiden, dürfte ihre Heterogenität auf unterschiedlichem Gehalt an Sialinsäure (29) und Kohlenhydraten beruhen.

Diese Ursache wurde bereits für das Auftreten eigenständiger Formen der alkalischen Phosphatase (20) und Arylamidase (21) im Serum postuliert, da sich diese Enzyme in ihrer elektrophoretischen Beweglichkeit nicht durch Triton-, sondern durch NeuraminidaseBehandlung verändern. Offenbar modifizieren Membran-Glykosyltransferasen das Enzymmolekül beim Übertritt in den Intravasalraum so nachhaltig, daß die Lectinaffinität der zirkulierenden Formen keine diagnostische Zuordnung zum Ursprungsort mehr erlaubt.

\section{Danksagung}

Mein besonderer Dank gilt Frau Barbara Flatter für ihre ausgezeichnete technische Assistenz bei allen Untersuchungen.

\section{Literatur}

1. Lorentz, K., Flatter, B. \& Kolle, F. W. (1979) diese Z. 17, 757-765.

2. Richterich, R. (1971) Klinische Chemie, 3. Aufl., S. 305309, Verlag S. Karger, Basel.

3. Lorentz, K., Koch, C.-D., Flatter, B. \& Molz; J. (1975) diese $2.13,49-52$.

4. Hausamen, T. U., Helger, R., Rick, W. \& Gross, W. (1967) Clin. Chim. Acta 15, 241-245.

5. Szasz, G., Weimann, G., Stähler, F., Wahlefeld, A. W. \& Persijn, J. P. (1974) diese Z. 12, 228.

6. Knedel, M. \& Böttger, R. (1967) Klin. Wochenschr. 45, 325-327.

7. Lorentz, K., Flatter, B. \& Augustin, E. (1979) Clin. Chem. $25,1714-1720$.

8. Lorentz, K. \& Flatter, B. (1977) diese Z. 15, 101-108.

9. Patel, S. \& O'Gorman, P. (1973) Clin. Chim. Áçta 49, 11-17. 
10. Schmidt, E. \& Schmidt, F. W. (1970) Enzymol. Biol. Clin. 11, 67-129.

11. Huseby, N.-E. (1977) Biochim. Biophys. Acta 483, 46-56.

12. Shaw, L. M., London, J. W. \& Petersen, L. E. (1978), Clin. Chem. 24, 905-915.

13. Shaw, L. M., Petersen-Archer, L., London, J. W. \& Marsh, E. (1980), Clin. Chem. 26, 1523-1527.

14. Komoda, T. \& Sakagishi, Y. (1976) Biochim. Biophys. Acta $438,138-152$.

15. Dubach, U. C. \& Schmidt, U. (1970) Enzymol. Biol. Clin. $11,32-51$.

16. Tate, S. S. \& Ross, M. E. (1977) J. Biol., Chem. 252, 6042-6045.

17. Komoda, T. \& Sakagishi (1976) Biochim. Biophys. Acta $445,645-660$.

18. Greenberg, E., Wollaeger, E. E., Fleisher, G. A. \& Engstrom, G. W. (1967) Clin. Chim. Acta 16, 79-89.

19. Delbrück, A. (1970) Enzymol. Biol. Clin. 11, 130-153.

20. Lorentz, K., Flatter, B. \& Heydrich, D. (1974) diese Z. $12,81-86$.

21. Lorentz, K., Marunowski, A. \& Ritter, U. (1974) diese Z. $12,468-473$

22. Peters, B. P., Ebisu, S., Goldstein, I. J. \& Flashner, M. (1979) Biochemistry 18, 5505-5511.

23. Monsigny, M., Roche, A.-C., Sene, C., Maget-Dana, R. \& Delmotte, F. (1980) Eur. J. Biochem. 104, 147-153.

24. Greenaway, P. J. \& LeVine, D. (1973) Nature (London) New Biol. 241, 191-192.
25. Köttgen, E. \& Gerok, W. (1976) Klin. Wochenschr. 54, 439-444.

26. Shaw, L. M. \& London, J. W. (1979) Clin. Chem. 25, 1155.

27. Shaw, L. M. \& Petersen-Archer, L. (1979) Clin. Biochem. $12,256-260$.

28. Lehmann, F.-G. (1980), Klin. Wochenschr. 58, 947-951.

29. Huseby, N.-E. (1981) Clin. Chim. Acta 111, 39-45.

30. Sidorowicz, W., Hsia, W.-Ch., Maslej-Zownir, O. \& Behal, F. J. (1980) Clin. Chim. Acta 107, 245-256.

31. Dulaney, J. T. (1979) Anal. Biochem. 99, 254-267.

32. Debray, H., Decout, D., Strecker, G., Montreuil, J. \& Monsigny, M. (1979) Prot. Biol. Fluids 27, 451-454.

33. Higashino, K., Otani, R., Kudo, S. \& Yamamura, Y. (1977) Clin. Chem. 23, 1615-1623.

34. Komoda, T. \& Sakagishi, Y. (1978) Biochim. Biophys. Acta 523, 395-406.

35. Chang, C.-H., Angellis, D. \& Fishman, W. H. (1975) Mol. Cell. Biochem. 9, 55-57.

36. Echetubu, Z. O.\& Moss, D. W. (1979) Clin. Chim. Acta $95,433-441$.

37. Huseby, N.-E. (1978) Biochim. Biophys. Acta 522, $354-362$.

38. Tsuji, A., Matsuda, Y. \& Katunuma, N. (1980) Clin. Chim. Acta 104, 361-366.

39. Tsuchida, S., Imai, F. \& Sato, K. (1980), J. Biochem. (Tokyo) 89,775-782.
Prof. Dr. Klaus Lorentz Kronsforder Allee 71-73 D-2400 Lübeck 
